\title{
Aetiology and antifungal susceptibility of yeast bloodstream infections in a Hungarian university hospital between 1996 and 2000
}

\author{
ILONA DÓCZI, ERIKA DÓSA, EDIT HAJDÚ and ELISABETH NAGY \\ Institute of Clinical Microbiology, Medical Faculty, University of Szeged, Szeged, Hungary
}

\begin{abstract}
The purpose of this study was to evaluate the aetiology and susceptibility of different Candida species originating from blood cultures received from different clinical wards of the University Hospital in Szeged, Hungary, from 1996 to 2000. A total of 145 episodes of fungaemia occurred in 68 patients. In $73.5 \%$ of the patients the infections were due to Candida albicans, $7.3 \%$ to $C$. parapsilosis, $5.9 \%$ to $C$. krusei, $4.4 \%$ to $C$. tropicalis and $3 \%$ each to $C$. glabrata, other Candida spp. and Cryptococcus neoformans. There were no appreciable differences in the distribution of yeast species during the 5-year period: C. albicans remained the predominant species causing bloodstream infections in this hospital, similar to the results of other studies (Norway, SENTRY Program in USA, Canada and South America). Most of the Candida isolates (39.3\%) were from blood cultures of patients hospitalised in surgical wards, $28.3 \%$ were from adult intensive care units (ICUs), $13.8 \%$ from paediatric ICUs, $11 \%$ from haematology and $7.6 \%$ from cardiology departments. MICs for amphotericin B, fluconazole and itraconazole were determined for $83 \%$ of the isolates. All isolates were susceptible to amphotericin $B$. The percentage of yeast isolates with decreased susceptibility or resistance to fluconazole was smaller $(15.7 \%)$ than that for itraconazole $(24 \%)$.
\end{abstract}

\section{Introduction}

Yeasts are emerging as important aetiological agents of nosocomial infections and infections in immunocompromised patients [1-4]. Colonisation of the gastrointestinal tract and other body sites is likely to be the initial step preceding systemic yeast invasion.

Blood cultures can be used to diagnose invasive candida infections [5]. Besides conventional methods, several fully automated blood culture systems (Bact/Alert, Organon Technica; Bactec, Becton Dickinson; Vital, bioMérieux) have recently become available for the isolation of fungi. Despite the fact that blood cultures remain the basic tool for the diagnosis of fungaemia, this method may fail to detect as many as $50 \%$ of disseminated candidosis cases. The concomitant colonisation of mucosal surfaces by the same Candida species may be the only laboratory sign of a systemic fungal infection in immunocompromised patients [6-8].

Received 28 Sept. 2001; revised version accepted 14 Feb. 2002.

Corresponding author: Professor E. Nagy (e-mail: nagye@ mlab.szote.u-szeged.hu).
Candida spp. should be identified to species level, as some of the non-albicans Candida spp. have proved to be resistant to antimycotic agents, such as azoles, used in the empirical therapy of invasive fungal infection $[9,10]$. In bloodstream infections, the breakpoints or MICs of antifungal agents must be determined to identify risk factors for infections by resistant yeasts and to assess the clinical relevance of the treatments. Similar reports in Europe have shown that bloodstream infections caused by yeasts are increasing [11-13].

The aims of this study were to establish the occurrence of different Candida spp. originating from blood cultures received from different clinical wards of the University Hospital in Szeged, Hungary, during a 5-year period. The presence of the same species within a 1-week interval in samples other than blood cultures was also evaluated. The susceptibility of these isolates to antifungal agents and the aetiology of bloodstream infections were also evaluated in this retrospective study.

\section{Materials and methods}

The automated Vital blood culture system (bioMérieux, 
Marcy-1'Etoile, France) was used to diagnose bloodstream infections. Aerobic and anaerobic blood culture bottles were used in parallel for all patients. In accordance with the manufacturer's instructions, separate blood culture bottles were not used in the event of the suspicion of candidaemia. If a positive signal was noticed, Gram's or acridine orange staining, or both, was done. All positive bottles in which yeasts were detected by direct microscopy were subcultured on CHROM agar (Becton-Dickinson, Budapest, Hungary) for direct isolation of the yeasts, in parallel with Sabouraud agar and Columbia blood agar. C.albicans, C. glabrata, C. tropicalis and C. krusei were identified on the basis of the typical colours of their colonies on CHROM agar. All other species were identified with ATB 32C (bioMérieux).

The in-vitro susceptibilities of various Candida isolates to antifungal agents were determined by the Etest method (AB Biodisk, Solna, Sweden) [14]. This was performed in accordance with the manufacturer's instructions with the use of RPMI media. The drug concentration ranges used were $0.016-256 \mathrm{mg} / \mathrm{L}$ for fluconazole, and $0.002-32 \mathrm{mg} / \mathrm{L}$ for itraconazole and amphotericin B. The MICs for amphotericin B were taken as the drug concentrations causing 95\% inhibition. MICs for azoles were read at the visually selected end-points of $80 \%$ inhibition of growth [14]. Interpretative susceptibility criteria for fluconazole, itraconazole and amphotericin B were used as published by the NCCLS [15].

\section{Results}

During the 5-year study period (1996-2000), the number of blood cultures in this 1240-bed university hospital varied between 6762 and 8845 per year (Table 1). The overall percentage of positive blood cultures was 14.5$18.8 \%$, with little year-to-year variation and with 1.4 $2.6 \%$ of all positive blood cultures yielding yeasts.

The 143 yeast isolates among a total of 145 isolates obtained from bloodstream infections during this period were identified to species level (Table 2): 112 (77.2\%) isolates were C. albicans, $3(2.1 \%)$ C. glabrata, 13 (9\%) C. krusei, 7 (4.8\%) C. parapsilosis, 5 (3.4\%) C. tropicalis, 2 (1.4\%) Candida spp. and 3 (2.1\%)
Cryptococcus neoformans. In some cases, the same species was isolated from more than one blood culture bottle from the same patient. The distribution of the species isolated from the positive blood cultures and the distribution of the species among separate episodes of candidaemia did not differ markedly (Table 2).

Table 3 details the distribution of these isolates in the various departments. Most yeast isolates (57, 39.3\%) were from the blood cultures of patients in surgical wards. Fewer isolates were found in blood cultures from patients in adult intensive care units (ICUs) $(41,28.3 \%)$ and $20(13.8 \%)$ were from paediatric ICUs. Relatively few isolates were from patients hospitalised in haematology $(16,11 \%)$ or cardiology $(11,7.6 \%)$ departments. Altogether, 68 patients had a bloodstream infection caused by yeasts during this period: $45.6 \%$ of all episodes occurred in patients hospitalised in ICUs and $16.2 \%$ of the cases were diagnosed among patients with haematological disorders. Two patients had $\mathrm{Cr}$. neoformans sepsis: one was an immunosuppressed patient from the paediatric ICU and one had received long-term treatment with high-dose steroids because of dermatomyositis. (Differences between species distributions and departments were not statistically significant by the $\chi^{2}$ test, $\mathrm{p}>0.05$.)

Of the 68 patients, 26 had yeast only in their blood cultures. In the other 42 patients, the same yeast was also found in other samples: 19 patients in one other sample (urogenital tract, faeces, respiratory tract or wound), 12 in two other samples (gastrointestinal-urogenital tracts, CVC-respiratory tract, CVC-urogenital tract, urogenital-respiratory tracts, wound-respiratory tract, wound-urogenital tract or CSF-respiratory tract), 10 in three other samples (CVCgastrointestinal-respiratory tracts, CVC-gastrointestinalurogenital tracts, gastrointestinal-urogenital-respiratory tracts, CVC-urogenital-respiratory tracts, CVC-woundurogenital tract, wound-urogenital-respiratory tracts or CSF-wound-urogenital tract) and 1 patient in 4 other samples (CVC-wound-urogenital-respiratory tracts) besides the blood cultures. (Only samples taken within 1 week were considered.)

The susceptibilities of the blood culture isolates were evaluated retrospectively. Before 1997, resistance was not determined routinely for yeasts isolated from systemic infections. After 1997, isolates were tested

Table 1. Blood culture results at the University Hospital in Szeged between 1996 and 2000

\begin{tabular}{|c|c|c|c|c|c|}
\hline \multirow[b]{2}{*}{ Year } & \multicolumn{2}{|c|}{ Blood culture bottles } & \multicolumn{3}{|c|}{ Positive bottles with } \\
\hline & tested & $\begin{array}{c}\text { positive } \\
\text { (\% of all tested) }\end{array}$ & $\begin{array}{l}\text { aerobic } \\
\text { bacteria }\end{array}$ & $\begin{array}{c}\text { anaerobic } \\
\text { bacteria }\end{array}$ & $\begin{array}{c}\text { yeasts } \\
\text { (\% of all positive) }\end{array}$ \\
\hline 1996 & 6782 & $1074(15.9)$ & 1024 & 26 & $24(2.2)$ \\
\hline 1997 & 8569 & $1612(18.8)$ & 1539 & 31 & $42(2.6)$ \\
\hline 1998 & 8759 & $1290(14.7)$ & 1239 & 33 & $18(1.4)$ \\
\hline 1999 & 8845 & $1279(14.5)$ & 1226 & 22 & $31(2.4)$ \\
\hline 2000 & 8695 & $1266(14.6)$ & 1206 & 30 & $30(2.4)$ \\
\hline
\end{tabular}


Table 2. Species distribution of yeasts isolated from blood cultures between 1996 and 2000

\begin{tabular}{|c|c|c|c|c|c|c|}
\hline \multirow[b]{2}{*}{ Species } & \multicolumn{5}{|c|}{ Number of isolates (number of patients) } & \multirow{2}{*}{$\begin{array}{c}\text { Total number } \\
(\%) \text { of isolates, } \\
1996-2000\end{array}$} \\
\hline & 1996 & 1997 & 1998 & 1999 & 2000 & \\
\hline C. albicans & $14(12)$ & $40(10)$ & $7(5)$ & $25(11)$ & $26(12)$ & $112(77.2)$ \\
\hline C. glabrata & 0 & 0 & $2(1)$ & $1(1)$ & 0 & $3(2.1)$ \\
\hline C. krusei & $6(3)$ & 0 & $7(1)$ & 0 & 0 & $13(9)$ \\
\hline C. parapsilosis & $2(1)$ & $1(1)$ & 0 & $2(1)$ & $2(2)$ & $7(4.8)$ \\
\hline C. tropicalis & $1(1)$ & $1(1)$ & 0 & $3(1)$ & 0 & $5(3.4)$ \\
\hline Candida spp. & $1(1)$ & 0 & $1(1)$ & 0 & 0 & $2(1.4)$ \\
\hline Cr. neoformans & 0 & 0 & $1(1)$ & 0 & $2(1)$ & $3(2.1)$ \\
\hline Total & $24(18)$ & $42(12)$ & $18(9)$ & $31(14)$ & $30(15)$ & $145(100)$ \\
\hline
\end{tabular}

Table 3. Species distribution of yeasts isolated from different departments between 1996 and 2000

\begin{tabular}{|c|c|c|c|c|c|c|}
\hline \multirow[b]{2}{*}{ Species } & \multicolumn{5}{|c|}{ Number of isolates (number of patients) } & \multirow{2}{*}{$\begin{array}{c}\text { Total number (\%) } \\
\text { of episodes, } \\
1996-2000\end{array}$} \\
\hline & Cardiology & Haematology & Adult ICUs* & Pediatric ICUs ${ }^{\dagger}$ & Surgery & \\
\hline C. albicans & $9(3)$ & $10(8)$ & $37(16)$ & $16(8)$ & $40(15)$ & $50(73.5)$ \\
\hline C. glabrata & 0 & 0 & $2(1)$ & 0 & $1(1)$ & $2(2.9)$ \\
\hline C. krusei & 0 & $4(2)$ & 0 & 0 & $9(2)$ & $4(5.9)$ \\
\hline C. parapsilosis & $1(1)$ & $2(1)$ & $2(2)$ & 0 & $2(1)$ & $5(7.3)$ \\
\hline C. tropicalis & 0 & 0 & 0 & $2(2)$ & $3(1)$ & $3(4.4)$ \\
\hline Candida spp. & $1(1)$ & 0 & 0 & $1(1)$ & 0 & $2(2.9)$ \\
\hline Cr. neoformans & 0 & 0 & 0 & $1(1)$ & $2(1)$ & $2(2.9)$ \\
\hline Total & $11(5)$ & $16(11)$ & $41(19)$ & $20(12)$ & $57(21)$ & $68(100)$ \\
\hline
\end{tabular}

${ }^{*}$ Including surgical and internal ICUs.

${ }^{\dagger}$ Including neonatal and paediatric ICUs.

for their susceptibilities to amphotericin B, fluconazole and itraconazole (Table 4). All the yeasts tested (98 C. albicans, 3 C. glabrata, 7 C. krusei, 5 C. parapsilosis, 4 C. tropicalis, 1 Candida spp. and 3 Cr. neoformans) were susceptible to amphotericin B, with MICs $\leqslant 1 \mathrm{mg} / \mathrm{L}$. Among the C. albicans isolates, $89 \%$ and $79 \%$ were susceptible to fluconazole and itraconazole, respectively, $6 \%$ and $16 \%$ of them in a dose-dependent way; five of these isolates (5\%) were resistant to these antimycotic agents. Two $C$. glabrata isolates were susceptible to fluconazole and itraconazole. One $C$. glabrata isolate was susceptible to fluconazole in a dose-dependent way, but resistant to itraconazole. The seven C. krusei isolates that were resistant to itraconazole with an $\mathrm{MIC} \geqslant 1 \mathrm{mg} / \mathrm{L}$ were from one patient. All five C. parapsilosis isolates were susceptible to both drugs, as were all four C.tropicalis isolates and the one Candida sp. isolate. All three $C r$. neoformans isolates were susceptible to all antimycotic agents examined.

Table 4. Antifungal susceptibilities of yeasts isolated from blood cultures

\begin{tabular}{|c|c|c|c|}
\hline \multirow[b]{2}{*}{$\begin{array}{l}\text { Species } \\
\text { (number of isolates tested) }\end{array}$} & \multicolumn{3}{|c|}{ Number of isolates (\%) classified as indicated by fluconazole Etest } \\
\hline & Susceptible $(\mathrm{MIC} \leqslant 8 \mathrm{mg} / \mathrm{L})$ & $\begin{array}{l}\text { Susceptible dose-dependent } \\
\quad(\mathrm{MIC} \geqslant 16 \leqslant 32 \mathrm{mg} / \mathrm{L})\end{array}$ & Resistant $(\mathrm{MIC} \geqslant 64 \mathrm{mg} / \mathrm{L})$ \\
\hline C. albicans (98) & $87(88.8)$ & $6(6.1)$ & $5(5.1)$ \\
\hline C. glabrata (3) & $2(66.7)$ & $1(33.3)$ & 0 \\
\hline C. krusei (7) & 0 & 0 & $7(100)$ \\
\hline C. parapsilosis (5) & $5(100)$ & 0 & 0 \\
\hline C. tropicalis (4) & $4(100)$ & 0 & 0 \\
\hline Candida spp. (1) & $1(100)$ & 0 & 0 \\
\hline \multirow[t]{3}{*}{ Cr. neoformans (3) } & $3(100)$ & 0 & 0 \\
\hline & \multicolumn{3}{|c|}{ Number of isolates $(\%)$ classified as indicated by itraconazole Etest } \\
\hline & Susceptible $(\mathrm{MIC} \leqslant 0.125 \mathrm{mg} / \mathrm{L})$ & $\begin{array}{l}\text { Susceptible dose-dependent } \\
(\mathrm{MIC} \geqslant 0.25 \leqslant 0.5 \mathrm{mg} / \mathrm{L})\end{array}$ & Resistant $(\mathrm{MIC} \geqslant 1 \mathrm{mg} / \mathrm{L})$ \\
\hline C. albicans (98) & $77(78.6)$ & $16(16.3)$ & $5(5.1)$ \\
\hline C. glabrata (3) & $2(66.7)$ & 0 & $1(33.3)$ \\
\hline C. krusei (7) & 0 & 0 & $7(100)$ \\
\hline C. parapsilosis (5) & $5(100)$ & 0 & 0 \\
\hline C. tropicalis (4) & $4(100)$ & 0 & 0 \\
\hline Candida spp. (1) & $1(100)$ & 0 & 0 \\
\hline Cr. neoformans (3) & $3(100)$ & 0 & 0 \\
\hline
\end{tabular}




\section{Discussion}

This retrospective study evaluated the occurrence of bloodstream infections caused by yeasts among patients hospitalised in different clinical wards of the University Hospital of Szeged. As compared with international studies (5-10 infections per 10000 admissions [16]), overall fewer bloodstream infections were caused by Candida spp. during this period, with a rate of 2-4.1 per 10000 admissions. Much higher rates are observed when surgical ICUs (9.8 per 1000 admissions) or neonatal ICUs (12.3 per 1000 admissions) are evaluated separately [17]. However, a recent epidemiological survey on candidaemia in Europe, involving four countries and 76 hospitals, revealed a rate of $0.1-$ 4.3 per 10000 patients hospitalised for more than 1 day [18].

Several studies from different parts of the world have shown that the number of bloodstream infections caused by yeasts is increasing. The SENTRY Antimicrobial Surveillance Program confirmed that Candi$d a$ spp. remain the fourth most common cause of nosocomial bloodstream infections, but the frequency may vary widely in different institutions [19].

There have been similar reports in Europe. Studies from a Danish university hospital showed a gradual increase in the annual incidence of fungaemia from 1989 to 1994 [11]. During the study period the most frequently isolated yeast in Hungary was $C$. albicans $(77.2 \%)$, which is similar to results in other European (54-66\%) and American (45-55\%) countries [13, 1921]. The highest rate of $C$. albicans isolates in any country was reported from Hungary. The highest numbers of isolates of non-albicans Candida spp. were C. glabrata in Norway and the USA, C. parapsilosis in the Slovak Republic, Italy, Canada and Latin America, and C. krusei in Hungary. With respect to the number of episodes (Table 3), C. parapsilosis was the second most frequent non-albicans Candida sp. causing bloodstream infections, with 5 episodes out of 68 . There were more $C$. glabrata than $C$. krusei isolates in America, but this rate varies widely in Europe [13, 1921].

Bloodstream infections caused by yeasts usually originate from the gastrointestinal tract in immunocompromised patients. Risk factors for candida bloodstream infections include colonisation with the organism in other body sites associated with the presence of intravascular catheters. Carriage of the infecting strain on the hands of health-care workers may also cause nosocomial infections [22]. In patients who have candidaemia, the same species may be isolated from specimens other than blood cultures at the same time (within 1 week), and colonisation of different body sites may be the first sign of a systemic candida infection. Among the 68 patients whose blood cultures proved the existence of candidaemia, 42 were colonised with the same species in other specimens. The most frequent sites of colonisation with the same Candida sp. were the urogenital tract, the CVC and the respiratory tract. The colonisation in the different body sites was naturally influenced by various factors, such as the location of hospitalisation, the underlying disease and previous antibiotic treatment.

The clinical outcome in these yeast-infected patients was also evaluated retrospectively in this study. Of 50 patients infected by C. albicans, $23(46 \%)$ died as a result of bloodstream infection and 16 (32\%) survived. (Information concerning the outcome of 11 patients is not available.) Among patients with bloodstream infection due to non-albicans Candida spp., the lowest mortality rate was in cases of $C$. tropicalis: two patients $(66.7 \%)$ survived and one $(33.3 \%)$ died. The second lowest mortality rate was in $C$. parapsilosis bloodstream infection: three $(60 \%)$ patients survived and two (40\%) died. The highest mortality was due to C. krusei: all four infected patients died. Each patient infected by C. glabrata (two patients) or other Candida spp. (two patients) died from their bloodstream infections. Two patients with $\mathrm{Cr}$. neoformans infections survived. In summary, $40 \%$ of these patients are known to have survived their bloodstream infections caused by yeasts. Differences relating to outcome and yeast species $(C$. albicans vs. non-albicans Candida spp.) were not statistically significant $\left(\chi^{2}\right.$ test, $\left.\mathrm{p}>0.05\right)$.

In conclusion, these results demonstrate that there were no statistically significant differences in the distribution of these species during the 5-year period studied. $C$. albicans remained the predominant species causing bloodstream infections in this hospital. In comparison with international reports [16], the number of bloodstream infections caused by yeasts per 10000 admissions was smaller in the present study. However, there are similar data from other European surveys [18]. The susceptibilities to amphotericin B and azoles are variable, but remain in the normal range.

As far as we are aware, this is the first report on the prevalence, species distribution and resistance rate of yeasts causing bloodstream infections in Hungary.

This study was supported in part by Hungarian research grant OTKA (TO 23652). We thank Pfizer for supplying the Etests. We also thank Maria Kakuja MD, Krisztina Mader MD and other colleagues for the data collection. Part of this study was included in an epidemiological surveillance of candidaemia in Europe, organised by R. Grillot.

\section{References}

1. Beck-Sague C, Jarvis WR. Secular trends in the epidemiology of nosocomial fungal infections in the United States, 19801990. National Nosocomial Infections Surveillance System. J Infect Dis 1993; 167: 1247-1251.

2. Berenguer J, Fernández-Baca V, Sánchez R, Bouza E. In vitro activity of amphotericin B, flucytosine and fluconazole against yeasts causing bloodstream infections. Eur J Clin Microbiol Infect Dis 1995; 14: 362-365. 
3. Martín-Mazuelos E, Gutiérrez MJ, Aller AI et al. A comparative evaluation of Etest and broth microdilution methods for fluconazole and itraconazole susceptibility testing of Candida spp. J Antimicrob Chemother 1999; 43: 477-481.

4. Nagy E. Changing epidemiology of systemic fungal infections and the possibilities of laboratory diagnostics. Acta Microbiol Immunol Hung 1999; 46: 227-231.

5. Procop GW, Cockerill FR, Vetter EA, Harmsen WS, Hughes JG, Roberts GD. Performance of five agar media for recovery of fungi from isolator blood cultures. J Clin Microbiol 2000; 38: $3827-3829$.

6. Vincent J-L, Anaissie E, Bruining $\mathrm{H}$ et al. Epidemiology, diagnosis and treatment of systemic Candida infection in surgical patients under intensive care. Intensive Care Med 1998; 24: 206-216.

7. Dalle F, Franco N, Lopez J et al. Comparative genotyping of Candida albicans bloodstream and nonbloodstream isolates at a polymorphic microsatellite locus. J Clin Microbiol 2000; 38: 4554-4559.

8. Luu LN, Cowen LE, Sirjusingh C, Kohn LM, Anderson JB. Multilocus genotyping indicates that the ability to invade the bloodstream is widespread among Candida albicans isolates. $J$ Clin Microbiol 2001; 39: 1657-1660.

9. Rex JH, Page PW, Paetznick VL, Lozano-Chiu M, EspinelIngroff A, Anaissie EJ. Optimizing the correlation between results of testing in vitro and therapeutic outcome in vivo for fluconazole by testing critical isolates in a murine model of invasive candidiasis. Antimicrob Agents Chemother 1998; 42: $129-134$.

10. Meis JFGM, Verweij PE. Current management of fungal infections. Drugs 2001; 61 Suppl 1: 13-25.

11. Bruun B, Westh H, Stenderup J. Fungemia: an increasing problem in a Danish university hospital 1989 to 1994. J Clin Microbiol Infect 1995; 1: 124-126.

12. Voss A, Kluytmans JA, Koeleman JG et al. Occurrence of yeast bloodstream infections between 1987 and 1995 in five Dutch university hospitals. Eur J Clin Microbiol Infect Dis
1996; 15: 909-912.

13. Sandven P, Bevanger L, Digranes A et al. Constant low rate of fungemia in Norway, 1991 to 1996. J Clin Microbiol 1998; 36: 3455-3459.

14. Etest technical guide No. 4: Antifungal susceptibility testing of yeasts. Solna, Sweden, AB Biodisk.

15. National Committee for Clinical Laboratory Standards. Reference method for broth dilution antifungal susceptibility testing of yeasts. Approved standard M27-A. Villanova, PA, NCCLS. 1997.

16. Wenzel RP, Porter WB. Epidemiology of Candida bloodstream infections in the hospital. Trends in Invasive Fungal Infections, Malta, 14-16 October, 1999.

17. Rangel-Frausto MS, Wiblin T, Blumberg HM et al. National epidemiology of mycoses survey (NEMIS): variations in rates of bloodstream infections due to Candida species in seven surgical intensive care units and six neonatal intensive care units. Clin Infect Dis 1999; 29: 253-258.

18. Grillot R. Epidemiological survey on candidemia in Europe. Mycology Newsletter 1998; 2: 11-13.

19. Pfaller MA, Diekema DJ, Jones $\mathrm{RN}$ et al. International surveillance of bloodstream infections due to Candida species: frequency of occurrence and in vitro susceptibilities to fluconazole, ravuconazole and voriconazole of isolates collected from 1997 through 1999 in the SENTRY Antimicrobial Surveillance Program. J Clin Microbiol 2001; 39: 3254-3259.

20. Krcmery V, Kovacicova G. Longitudinal 10-year prospective survey of fungaemia in Slovak Republic: trends in etiology in 310 episodes. Slovak Fungaemia study group. Diagn Microbiol Infect Dis 2000; 36: 7-11.

21. Luzzati R, Amalfitano G, Lazzarini L et al. Nosocomial candidemia in non-neutropenic patients at an Italian tertiary care hospital. Eur J Clin Microbiol Infect Dis 2000; 19: 602-607.

22. Levin AS, Costa SF, Mussi NS et al. Candida parapsilosis fungaemia associated with implantable and semi-implantable central venous catheters and the hands of health care workers. Diagn Microbiol Infect Dis 1998; 30: 243-249. 